\title{
MobAngelo: Assistência Emergencial em Ambientes urbanos
}

\author{
Lucas Braz Cunha ${ }^{1}$, Agnaldo de Souza Batista ${ }^{1}$, Aldri Santos ${ }^{1,2}$ \\ ${ }^{1}$ Núcleo de Redes Sem-Fio e Redes Avançadas (NR2) - PPGInf - UFPR \\ ${ }^{2}$ Departamento de Ciência da Computação - UFMG \\ \{1bc16, asbatista\}@inf.ufpr.br, aldri@dcc.ufmg.br
}

\begin{abstract}
The assistance to people in emergency health situations before medical care can contribute to their survival, such as in a respiratory arrest where response time is essential. In order to reduce the impact of the waiting time before an attendance, this paper presents the MobAngelo system, which connects mobile devices of people nearby so that, according to their health knowledge, they can assist each other in first aid until specialized care occurs. MobAngelo works in urban and sparse environments through the creation of temporary networks so that the user is aware of other nearby users who need help.
\end{abstract}

Resumo. O auxílio a pessoas em situações emergenciais de saúde antes do atendimento médico pode colaborar para sua sobrevivência, como por exemplo diante de uma parada respiratória onde o tempo de resposta torna-se fundamental. A fim de minimizar o tempo de espera até um atendimento, este artigo apresenta o sistema MobAngeld ${ }^{1}$ que conecta dispositivos móveis de pessoas próximas de acordo com seu conhecimento, de modo a auxiliar nos primeiros socorros até ocorrer o atendimento especializado. O MobAngelo atua em ambientes urbanos e esparsos, por meio da criação de redes temporárias, tal que o usuário tenha ciência de outros usuários próximos que necessitam de ajuda.

\section{Introdução}

O uso intenso da Internet e da computação móvel tem contribuído ao provimento de serviços online de transações comerciais à prestação de serviços com diversos propósitos. A partir dos avanços dos meios de transmissões e da evolução dos dispositivos móveis, novas maneiras de prestação de serviços podem ser exploradas ou mesmo consolidadas. Muitos desses serviços, devido à interatividade, demandam a criação e manutenção de infraestruturas de redes locais e globais dinâmicas para assegurar sua operação. No entanto, as características típicas de ambientes dinâmicos, como a falta de infraestrutura de rede e a mobilidade dos dispositivos, dificultam o controle da disseminação dos dados em diversos domínios de aplicação. Particularmente, as demandas por serviços de saúde em redes se intensificaram, devido a sua importância na vida das pessoas. Conhecidos como e-health, eles manuseiam dados pessoais sensíveis e críticos; e na entrega desses dados a segurança deve ser preservada, assim como do acesso não autorizado [Batista 2019].

Os serviços de $e$-health são estudados por suas diversas aplicações na relação entre saúde e tecnologia. Em [Kowatsch and et al. 2017] é avaliada a utilização de um aplicativo para smartphones com a finalidade de modificar positivamente os hábitos de seus

\footnotetext{
${ }^{1}$ Este trabalho tem apoio do CNPq processos \#436649/2018-7 e \#426701/2018-6.
} 
usuários. O estudo de [Künzler 2019] estende a análise avaliando como o disparo de notificações nos dispositivos pode afetar o uso do aplicativo. A Telemedicina, outro serviço do grupo $e$-health, foi oficializada pelo Conselho Federal de Medicina Brasileiro no ano de 2020, durante a pandemia de COVID-19 [CFM 2020]. Esse serviço oferece atendimento médico através de chamadas virtuais pelas quais os profissionais de saúde podem orientar ou encaminhar seus pacientes. Entretanto, ainda há a necessidade de sistemas que se adéquem à situações mais extremas como por exemplo a ausência de infraestrutura de rede em situações de catástrofe. O trabalho de [Álvarez 2020] propõe o uso de redes distribuídas para mitigar as consequências da ausência de infraestrutura. Além disso, nesse contexto também é possível utilizar redes ad hoc formadas por dispositivos móveis (smartphones), que auxiliariam nos primeiros momentos de uma situação de emergência.

Assim, as redes ad hoc sem fio podem naturalmente ser utilizadas para auxiliar no atendimento de casos de emergência individual em ambientes externos aos hospitais. Por exemplo, em caso de uma pessoa em parada cardíaca, segundo a Associação Americana do Coração (do inglês, American Heart Association - AHA) e a Associação Americana de Acidente Vascular Cerebral (do inglês, American Stroke Asssociation - ASA), para cada minuto que uma pessoa permanece sem a realização de Reanimação Cardiopulmonar (RCP), sua chance de sobrevivência reduz entre 7 a 10\%. Por outro lado, a execução imediata de RCP e desfibrilação com o equipamento adequado podem dobrar as chances de sobrevivência [AHA and ASA 2013]. Logo, uma disseminação de dados pessoais sensíveis rápida, segura e robusta em ambientes externos urbanos, podem agilizar no atendimento de pessoas diante de situações emergenciais de saúde. Nesses ambientes, a disseminação dos dados deveria observar o contexto e a urgência da informação evolvida para prover a sua disponibilidade e um atendimento adequado. Dessa maneira, a proximidade entre as pessoas e a ubiquidade dos smartphones na vida urbana possibilitam que sistemas automatizados agilizem o auxílio no atendimento nesses ambientes [Batista et al. 2020], ainda que a confiança para disseminação de dados sensíveis constitua um desafio.

Este trabalho apresenta o sistema MobAngelo para coordenar e assegurar a disseminação de dados sensíveis de maneira segura em ambientes dinâmicos não estruturados, suportando as tomadas de decisão diante de situações emergenciais de saúde em um ambiente Zero-Knowledge, onde não há interações passadas entre os dispositivos. $\mathrm{O}$ MobAngelo, executado em sistemas Android, emprega o conceito de confiança social e comunidades de interesse. Assim, a similaridade dos interesses dos usuários dos dispositivos estabelece a confiança social permitindo a formação de comunidades de interesse, e a disponibilidade do serviço. Através do atributo de competência das pessoas, a confiança social possibilita ao sistema garantir a confiabilidade do serviço ao disseminar a mensagem para a pessoa próxima mais qualificada, contribuindo para um melhor atendimento. Além disso, ao longo do tempo, os dispositivos redefinem suas comunidades a partir das mudanças nas suas vizinhanças pela entrada e saída de dispositivos do raio de alcance.

Este artigo está organizado da seguinte forma: A Seção 2 descreve a ferramenta MobAngelo e o funcionamento dos componentes. A Seção 3 apresenta a avaliação da ferramenta. A Seção 4 apresenta a conclusão e os trabalhos futuros.

\section{A Ferramenta MobAngelo}

O sistema MobAngelo estabelece uma rede de comunicação dinâmica e onipresente de suporte à pessoas que apresentem um evento crítico de saúde de modo inesperado 
em ambientes urbanos, que estejam desacompanhadas e exijam atenção. Ele cria a possibilidade de pessoas ao redor e desconhecidas ajudarem umas as outras, de acordo com a sua capacitação em saúde, em situações de emergência individual ou coletiva. Assim, elas podem antecipadamente auxiliar de alguma maneira até que os socorristas cheguem ao local, colaborando para atenuar possíveis sequelas resultantes da demora no atendimento. $\mathrm{O}$ MobAngelo baseia-se nos conceitos de contribuição colaborativa (Crowdsourcing), rede social baseada em localização, comunidades de interesse, sistemas de recomendação, e antecipação de dados sensíveis. A ferramenta tem como principais funcionalidades a construção e manutenção de informações de dispositivos vizinhos, a análise da confiança com relação aos seus vizinhos e o envio de mensagens de eventos críticos de saúde.

\subsection{Especificação técnica}

O MobAngeld ${ }^{2}$ foi desenvolvido para dispositivos smartphones que utilizam o sistema operacional Android. Ele foi implementado com as linguagens Kotlin e Java, que são as mais comuns para desenvolvimento de aplicações para essa plataforma, e recomendadas pela Google [Developers 2021], a mantenedora do sistema operacional. Além disso, a linguagem Kotlin oferece uma sintaxe mais concisa e segura, sem perder as bibliotecas e referências Java, devido à interoperabilidade das duas linguagens. Logo, o uso desses recursos facilita a compreensão de outros desenvolvedores para com o código fonte do MobAngeld ${ }^{3}$. O sistema é compatível com dispositivos que possuam sistema operacional Android a partir da versão 7.0 (apelidada Nougat). A tecnologia Bluetooth é utilizada na comunicação entre os dispositivos da rede devido a sua presença massiva em smartphones da atual geração.

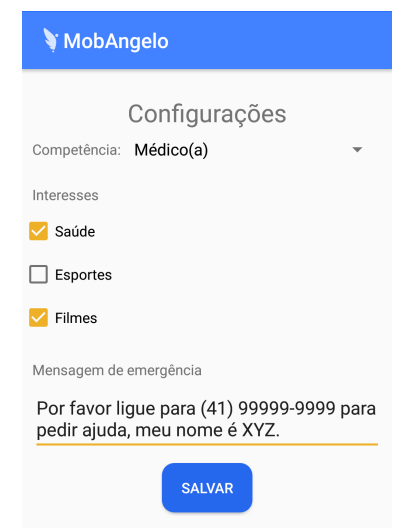

Figura 1. Configurações

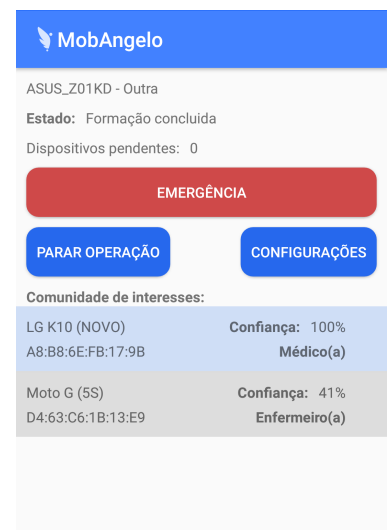

Figura 2. Operação

O usuário precisa configurar o MobAngelo informando sua competência em saúde e seus interesses, como ilustra a tela de Configuração na Figura 1. Além disso, o usuário deve pré-configurar a mensagem de emergência a ser enviada em caso de evento crítico. Nesse estágio de desenvolvimento, qualquer pessoa poderia usar o sistema, inclusive inserindo informações falsas. Porém, no caso da implantação do sistema para uso da população, a identificação e competência das pessoas poderiam ser validadas por uma entidade central, como um conselho de medicina, por exemplo. A competência e os interesses são utilizadas para o cálculo da confiança, explicado adiante. A competência refere-se à profissão ou habilidade do usuário e a sua relação com atividades em saúde. A Figura 2 exibe

\footnotetext{
${ }^{2}$ Código fonte e manual disponíveis em https://github.com/LucasBCunha/mob_angelo

${ }^{3}$ Licença Apache 2.0
} 
a tela operação do MobAngelo, permitindo acompanhar os dispositivos vizinhos que fazem parte da sua comunidade e, também, sinalizar um evento crítico através do botão "Emergência". Essa sinalização modifica o estado do sistema e envia um pedido de ajuda ao dispositivo próximo mais confiável.

\subsection{A Arquitetura do MobAngelo}

A arquitetura de implementação do MobAngeld ${ }^{4}$ baseia-se na arquitetura do modelo STEALTH ${ }^{5}$ (Social Trust-Based HEALTH Information Dissemination Control), proposta em [Batista 2019], e apresentada na Figura 3 em conjunto com suas características principais. Essa arquitetura possui dois módulos: $(i)$ Gestão de comunidades, que cria e realiza a manutenção das comunidades de interesse a partir dos dispositivos presentes na sua vizinhança, e computa a confiança com relação aos vizinhos; e $(i i)$ Gestão de eventos críticos, que controla e dissemina os dados em caso de emergência, escolhendo para qual dispositivo deve ser enviada uma mensagem de alerta.

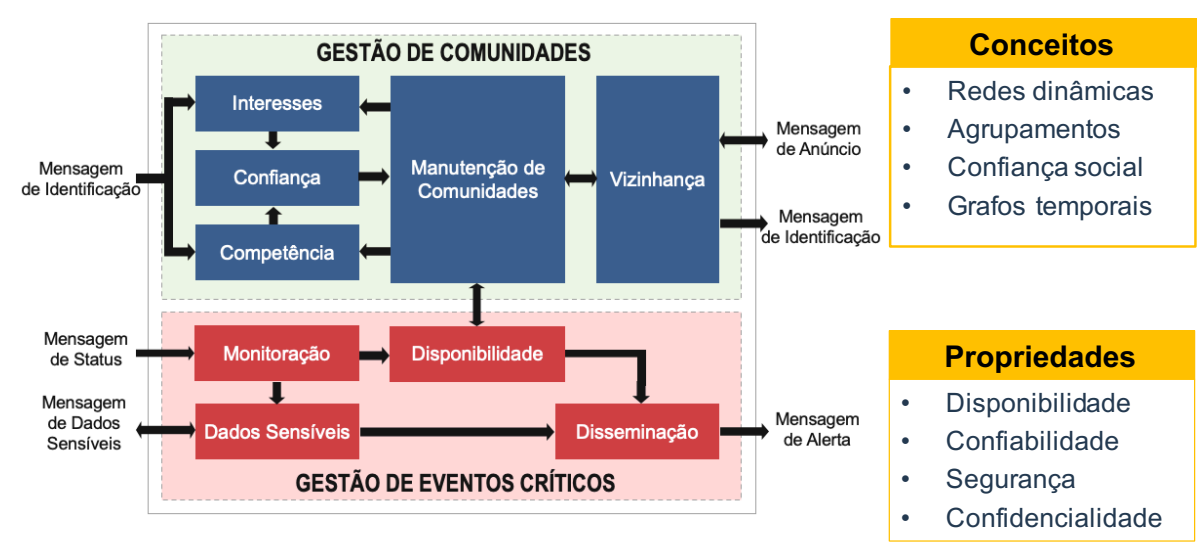

Figura 3. Arquitetura STEALTH e suas características

No desenvolvimento do MobAngelo, o módulo de Gestão de Comunidades, Figura 3 , baseia-se no conceito de comunidades de interesse (CoIs), quando um conjunto de dispositivos de rede agrupam-se pelas características comuns de seus portadores. $\mathrm{O}$ atributo social para agrupamento são os interesses dos portadores dos smartphones. Os interesses são assuntos que atraem o usuário, enquanto que os interesses em comum permitem estimar a afinidade entre pessoas nesses ambientes Zero-Knowledge. Em um ambiente externo, os usuários se movimentam e trocam mensagem entre si. De acordo com o alcance de cada dispositivo, eles interagem ao longo do tempo identificando os dispositivos próximos (i.e., vizinhos). As comunidades, por sua vez, são representações de configurações instantâneas da vizinhança ocorridas no ambiente no qual o dispositivo está inserido.

Para o envio de mensagens contendo dados sensíveis, o MobAngelo classifica os receptores para que apenas o conteúdo pertinente seja compartilhado e, também, que apenas o receptor desejado receba essa mensagem. Ele utiliza o conceito de confiança, que é a probabilidade estimada de que uma entidade exiba comportamento seguro para uma operação em determinada situação de incerteza [Cho et al. 2015], como parâmetro para

\footnotetext{
${ }^{4}$ Vídeo guia de instalação e utilização: https://youtu.be/14pu2xdE4kA

${ }^{5}$ Página do projeto: http://www.nr2.ufpr.br/asbatista/stealth.html
} 
suas decisões. A confiança é calculada entre dispositivos que fazem parte da mesma comunidade de interesses, através da similaridade dos seus interesses (afinidade) e da competência em saúde (conhecimento técnico) do proprietário do dispositivo avaliado. Ela é a medida que rege para qual dispositivo deve ser enviada a mensagem no caso de um evento crítico. Os dispositivos com alto grau de confiança são aqueles com maior nível de Competência em saúde e com mais Similaridade de interesses. O MobAngelo usa a taxonomia descrita no STEALTH que estabelece uma hierarquia de habilidades de saúde através de profissões relacionadas ao tema. Os valores de confiança relativos à competência $\left(T^{\text {Skill }}\right)$ variam no intervalo [0,1], sendo a competência de nível médico considerada a de valor máximo (1). A partir disso, a competência para outras profissões é calculada conforme sua distância em relação à competência médica na taxonomia estabelecida pelo STEALTH [Batista 2019]. As competências usadas pelo MobAngelo são um subconjunto da taxonomia original, descritas na Tabela 1 .

Tabela 1. Confiança atrib. às competências

\begin{tabular}{lc}
\hline Competência & Confiança \\
\hline Médico(a) & 1,00 \\
Técnico em enfermagem & 0,33 \\
Policial & 0,28 \\
Outra & 0,00 \\
\hline
\end{tabular}

$$
\begin{array}{r}
T_{i j}^{I}=\frac{\left|I_{i} \cap I_{j}\right|}{\left|I_{i}\right|} \\
T_{a b}=\frac{T_{a b}^{I}+T_{a b}^{S k i l l}}{2}
\end{array}
$$

A confiança é calculada com base na similaridade dos interesses entre o dispositivo avaliador $i$ e o dispositivo avaliado $j\left(T_{i j}^{I}\right)$ através da razão entre os interesses comuns de $i\left(I_{i}\right)$ e $j\left(I_{j}\right)$, e os interesses do dispositivo avaliador $i$, conforme apresentado na Equação 1 . A confiança em relação aos interesses é calculada somente se ambos os portadores - avaliado e avaliador - possuírem pelo menos o interesse em saúde. Portanto, o valor de $T_{i j}^{I}$ varia no intervalo ]0,1]. A confiança do dispositivo avaliador $i$ sobre o dispositivo avaliado $j\left(T_{i j}\right)$ é calculada pela soma da confiança em relação à competência de $i$ $\left(T_{i j}^{S k i l l}\right)$ e a confiança em relação aos seus interesses em comum $\left(T_{i j}^{I}\right)$, conforme Equação 2. Pressupondo que um dispositivo $i$ mensure a confiança $T_{i j}$ sobre um dispositivo $j$, cuja competência seja "Técnico em Enfermagem" e que ambos possuam apenas o interesse em saúde. Logo, pela Tabela 1, temos que $T_{i j}^{S k i l l}=0,33$. Assim, $T_{i j}^{I}=1$, visto que os dois dispositivos possuem interesses idênticos e, portanto, aplicando a Equação 2 , temos $T_{i j}=0,64$. Caso a competência de $j$ seja "Outra", $T_{i j}^{S k i l l}=0$, indicando que ele não possui habilidade de saúde. Como $T_{i j}^{I}=1$, a confiança de $i$ em $j$ terá o valor de 0,5.

O módulo Gestão de Eventos Críticos do MobAngelo, Figura3, dissemina os dados e garante o envio correto da mensagem de emergência. O componente Disponibilidade é responsável por encontrar a melhor pessoa para transmissão da mensagem, selecionando o dispositivo da comunidade de interesse com maior confiança. O componente Disseminação coordena o envio da mensagem ao dispositivo escolhido. O componente Monitoração é responsável pela integração com outros sistemas para monitorar o status de saúde do usuário. No contexto do MobAngelo, esse componente é simulado através do botão "Emergência", presente no aplicativo e ilustrado na Figura 2, O componente Dados sensíveis atua na seleção de quais dados devem ser enviados de acordo com a competência do dispositivo vizinho escolhido. O MobAngelo emprega uma mensagem padrão, que independe da competência do destinatário, conforme apresentado na Figura 1 . Ao receber ou enviar uma mensagem de emergência, a execução do módulo de gestão de comunida- 
des é interrompida, evitando que o dispositivo de uma pessoa em situação crítica receba dados sensíveis de outros dispositivos.

\subsection{Fluxo de comunicação}

Uma vez apresentados os dois módulos do MobAngelo, a partir do ponto de vista de um dispositivo, são descritas as rotinas executadas durante o seu ciclo de vida. Assim, após o inicio da execução do MobAngelo, sinalizado pelo usuário, o sistema inicia as rotinas de gestão das comunidades, responsáveis por recriar a comunidade de interesses a cada intervalo de aproximadamente 5 segundos, tendo tempo máximo de scan de 13 segundos. Primeiramente, executa-se um scan Bluetooth dos dispositivos que estão dentro do raio de cobertura. Em seguida, para cada dispositivo encontrado é estabelecida uma conexão Bluetooth, e essa comunicação deve ser respondida com os dados de identificação do outro dispositivo codificada como uma sequência de caracteres que respeite o formato competência; interesse 1, interesse 2 . A cada interação e identificação, o dispositivo descoberto é avaliado com base nos critérios explicados anteriormente e adicionado com sua confiança à comunidade de interesses. A Figura 4 ilustra um usuário - paciente - caminhando com o sistema MobAngelo ativo e durante seu trajeto interage com outros usuários formando a sua comunidade de interesse em saúde com participação daquelas pessoas que também possuem interesse em saúde: a médica, o técnico em enfermagem e o bombeiro. As comunidades são formadas somente pelos dispositivos que se encontram na área de cobertura, embora possam existir dispositivos Bluetooth que não façam parte da rede. Nesse caso, eles são ignorados após não responderem a mensagem de identificação ou em caso de recusa no estabelecimento da comunicação. Usuários que não possuem interesse em saúde também são excluídos na formação das comunidades, uma vez que a rede se propõe a facilitar e agilizar o auxílio no atendimento de saúde. Nesse caso, o dispositivo realiza o processo de identificação, mas seus dados são descartados pela contraparte. Essa situação é vista na Figura 4 pelo usuário de competência Outro (i.e., não possui qualquer competência na área de saúde) e também não possui interesse em saúde.

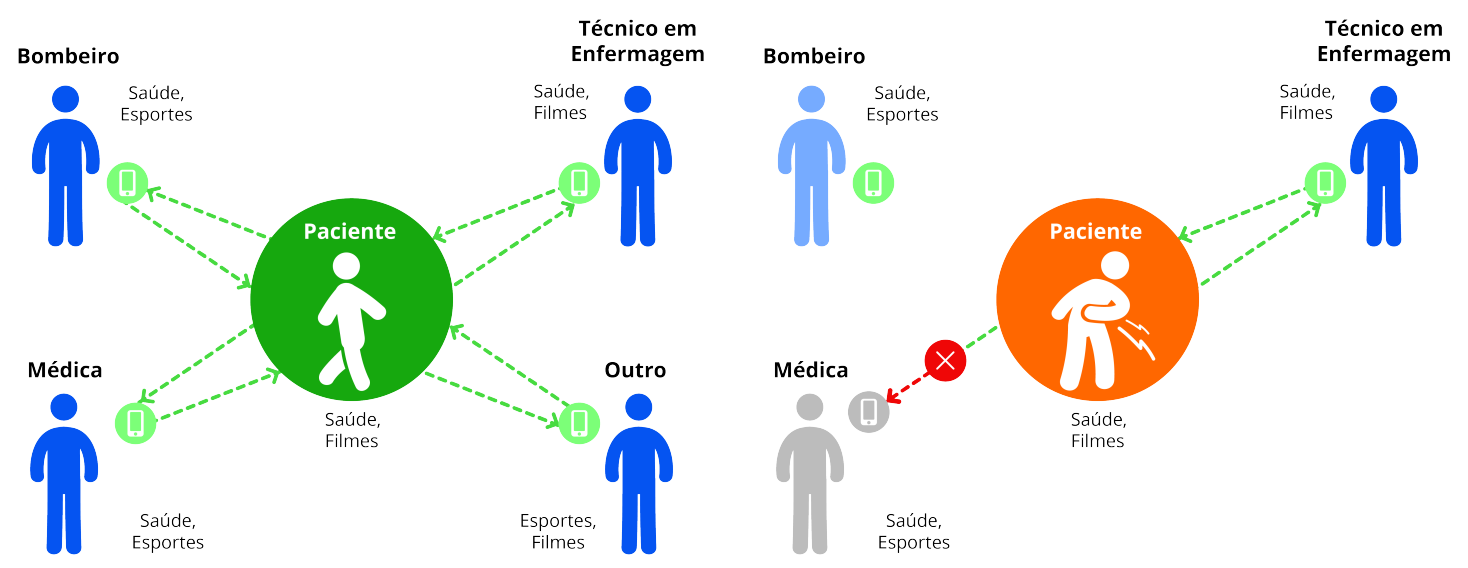

Figura 4. Formação da Col

Figura 5. Sinalização evento crítico

Diante da ocorrência de um evento crítico de saúde com o usuário, ele pode sinalizar que necessita de ajuda pressionando o botão Emergência disponível na tela principal do MobAngelo. Essa ação simula a integração com sensores externos que monitorem a saúde do usuário, por exemplo, um medidor de pressão ou de batimentos cardíacos. Ao 
receber esse sinal, o funcionamento do MobAngelo é alterado para priorizar o disparo da mensagem de evento crítico, tal que suas atividades de descoberta da vizinhança são interrompidas. A aplicação tenta, então, se conectar ao dispositivo melhor avaliado no quesito de confiança dentre aqueles que pertencem a sua comunidade de interesses vigente. Conforme demonstrado na Figura 1, a mensagem a ser enviada contém o texto que o usuário cadastrou e servirá como meio de identificação e direcionamento da ajuda, caso exista alguma condição preexistente que o usuário queira compartilhar (diabetes, pressão alta ou outras). Como visto na Figura 5, após o envio dessa mensagem para a médica, o MobAngelo do paciente aguarda a confirmação de seu recebimento para encerrar sua operação. Como o sistema não receba a confirmação dentro de um intervalo estabelecido, a mensagem é encaminhada ao segundo usuário mais confiável da sua comunidade de interesses - técnico em enfermagem. Caso o dispositivo desse usuário também não confirme o recebimento da mensagem do paciente, o processo seguirá sucessivamente até que a mensagem seja entregue ou que não existam vizinhos na sua comunidade de saúde.

\section{Avaliação}

Esta seção apresenta os resultados de uma avaliação de desempenho da comunicação efetuada pelo sistema. Os critérios analisados foram avaliados em um ambiente controlado com três dispositivos móveis como prova do conceito, pois devido à pandemia de COVID-19, a avaliação em um ambiente urbano com grande quantidade de usuários não foi possível. Entretanto, a partir desses três dispositivos é possível validar a efetividade da rede e analisar de maneira preliminar o seu desempenho. Para execução do experimento, os dispositivos utilizados possuíam versão 4.2 ou 4.0 da tecnologia Bluetooth com alcance de até 61 metros. Os dispositivos utilizados foram um smartphone Asus Zenfone 4 (Android 8.0), um Motorola G5s (Android 8.1) e um LG K10 (Android 7.0). Além dos dispositivos participantes, também havia no ambiente outro dispositivo Bluetooth que não fazia parte da rede, porém era visível durante os scans de outros dispositivos.

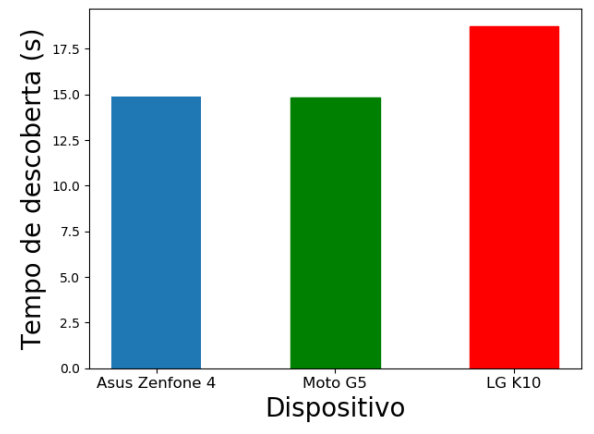

Figura 6. Duração média do scan de vizinhança

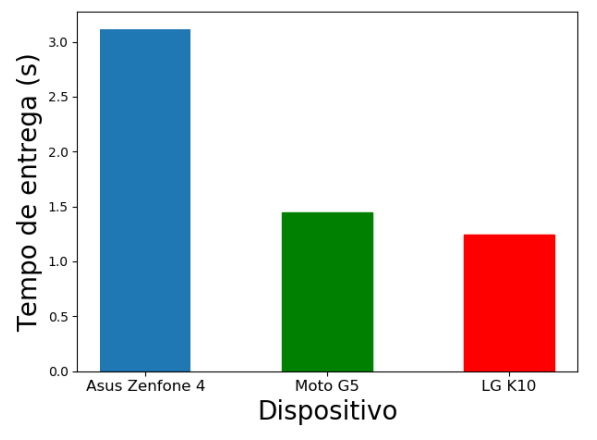

Figura 7. Tempo médio de entrega da mensagem de emergência

O experimento foi realizado duas vezes durante 15 minutos, tendo aproximadamente 10 interações de descoberta e construção da comunidade de interesses para cada dispositivo. A Figura 6 apresenta o tempo médio para construção da comunidade de interesses para cada dispositivo. Observa-se que os dois dispositivos com a mesma versão da tecnologia Bluetooth - Asus Zenfone 4 e Motorola G5s - tiveram um desempenho semelhante, com uma diferença inferior a 1 segundo. Contudo, o dispositivo LG K10, que possui uma versão inferior da tecnologia Bluetooth, 4.0, demorou em média 2 segundos 
a mais para a identificação e construção da sua comunidade de interesses. Observou-se também que em alguns casos dois dispositivos sincronizaram seu período de busca e acabaram não se reconhecendo enquanto mantiverem a operação, o que se provou ser um desafio para a execução do experimento. No segundo experimento, executado 5 vezes para cada dispositivo, avaliou-se o tempo para que os dispositivos disparem uma mensagem de emergência e recebam a confirmação, possibilitando que seja contabilizado o tempo total da entrega. Na Figura 7, temos a média do tempo para o envio da mensagem de evento crítico, originada pelo dispositivo nomeado, com destino aos 2 outros dispositivos presentes. Para o Zenfone 4, observou-se um grande intervalo com casos de $0.163 \mathrm{~s}$ a até $7.34 \mathrm{~s}$ de tempo total para envio. Isso aparenta ter relação com o tempo de espera pela confirmação devido ao estado de execução do dispositivo que recebeu a mensagem e também devido à falta de capacidade computacional dos outros 2 dispositivos.

\section{Conclusão e Trabalhos Futuros}

Este artigo apresentou o sistema MobAngelo, seus requisitos funcionais e um caso de uso. Trata-se de um aplicativo para dispositivos Android que não necessita de infraestrutura de rede para estabelecer sua comunicação. O sistema provê um meio de notificar pessoas dispostas a ajudar outras em situação de emergência de maneira colaborativa em ambientes urbanos. Ele garante a distribuição das informações sensíveis aos indivíduos mais capacitados e habilitados para recebê-las. Como trabalhos futuros implementaremos o cadastro e o envio de mensagens para cada nível de competência, o intervalo dinâmico entre scans Bluetooth e a integração com um serviço externo para validar a competência do usuário. Além disso, uma avaliação do consumo de energia pela aplicação.

\section{Referências}

AHA and ASA (2013). Every second counts: Rural and community access to emergency devices. https://www.heart.org/idc/groups/heart-public/@wcm/@adv/documents/ downloadable/ucm_301646.pdf. [Online] Acesso em: Mar. 2021.

Álvarez, F. (2020). Secure device-to-device communication for emergency response. $\mathrm{PhD}$ thesis, Technische Universität Darmstadt, Darmstadt.

Batista, A. (2019). Disseminação Segura de Dados Pessoais Vitais Para Apoio às tomadas de decisão em situações emergenciais. Master's thesis, UFPR, Curitiba, PR.

Batista, A., da Silva, G., de Lima, M., and dos Santos, A. (2020). Coordenação de múltiplos eventos críticos de saúde por redes dinâmicas de interesses sociais. In SBRC XXXVIII, pages 477-490, Porto Alegre, RS, Brasil. SBC.

CFM (2020). OFÍCIO CFM No1756/2020. https: / / portal. cfm.org.br/images/PDF/2020_ oficio_telemedicina.pdf. [Online] Acesso em: Maio 2021.

Cho, J.-H., Chan, K., and Adali, S. (2015). A survey on trust modeling. ACM Computing Surveys (CSUR), 48(2):1-40.

Developers, G. (2021). Develop android apps with kotlin. https://developer.android.com/ kotlin. [Online] Acesso em: Mar. 2021.

Kowatsch, T. and et al. (2017). Design and Evaluation of a Mobile Chat App for the Open Source Behavioral Health Intervention Platform MobileCoach. In Maedche, A., vom Brocke, J., and Hevner, A., editors, Designing the Digital Transformation, pages 485-489, Cham. Springer International Publishing.

Künzler, F. (2019). Context-aware notification management systems for just-in-time adaptive interventions. In 2019 IEEE International Conference on Pervasive Computing and Communications Workshops, pages 435-436. 